\title{
el empleo de la GRANA-CEMENTO en los firmes de carretera
}

\author{
OLEGARIO LLAMAZARES GOMEZ, Dr. ingeniero de caminos, canales y puertos
}

\begin{abstract}
Sinopsis Las capas de grava-cemento se emplean, desde hace varios años, en los firmes de carretera, sustituyendo a las bases granulares en los tramos en que el tráfico requiere secciones estructurales de elevada resistencia.

La experiencia ha aconsejado la evolución de esta técnica para llegar a capas de mayor calidad y garantía, con una selección más cuidada de los áridos en granulometría y forma, dosificaciones y tipo de cemento y condiciones de fabricación, puesta en obra y curado. En el presente artículo se informa sobre las actuales tendencias en todos estos puntos, indicando las exigencias de los Pliegos contractuales.
\end{abstract}

\section{antecedentes}

Las capas de grava-cemento han constituido una solución frecuente para bases de firmes de calidad en los últimos 10 años en que el tráfico pesado fue creciendo en intensidad y cargas por eje, haciendo insuficientes las secciones estructurales, integradas en gran parte por capas granulares.

En nuestras autopistas y otras vías de gran tráfico se aplica el tratamiento de cemento incluso a la sub-base, lo que presenta la doble ventaja: de conseguir una capa de mayor resistencia, así como, gracias a la corrección del ligante hidráulico, utilizar materiales locales que no serían adecuados en su estado natural, o sea, empleados con una simple estabilización mecánica.

El origen de la grava-cemento corresponde a las mezclas de granulometría gruesa utilizadas en el Estado de California. Sobre esta técnica se basaron las normas francesas de tan profusa aplicación, pues en el país vecino las capas de grava-cemento constituyen una solución generalizada tanto para secciones estructurales de nuevos firmes como para refuerzo de los existentes. Tales normas han sido objeto de evolución. llegándose a soluciones más perfeccionadas que se definen en una Orden circular del Ministère de l'Équipement cuya referencia consignamos (1).

En Inglaterra, el equivalente de estas capas de grava-cemento son las llamadas Lean concrete bases o bases de hormigón pobre, que van a emplearse en España en la autopista Bilbao-Behovia. De esta técnica peculiarmente inglesa nos hemos ocupado en una publicación anterior, a la que remitimos al lector (2).

Por lo que respecta a España, el empleo de grava-cemento se inició en la autopista Las Rozas-Villalba, donde nuestro compañero Frutos Santiago puso a punto esta nueva técnica por un imperativo de seguridad a la vista de que la aplicación de la viga de Benkelman, en los primeros tramos construidos, registró deflexiones superiores a las críticas.

El creciente tráfico del tramo, ya con una IMD de 8.000 crecerá con la intensidad de tráfico del tramo en cuestión, vehículos en 1964, con un tercio de vehículos pesados, y de éstos, la mitad con cargas por eje superiores a las máximas autorizadas, obligaba a una gran cautela, que como medida preventiva aconsejó prescindir de la base granular sensible a los cambios de humedad que daría lugar al agrietado y posterior degradación del firme. El huso granulométrico que se adoptó para la grava-cemento cumplía las recomendaciones francesas. Al efecto de determinar la proporción de cemento se dibujaron curvas de humedad-densidad para la energía del ensayo de compactación AASHO modificado. Las proporciones de ligante

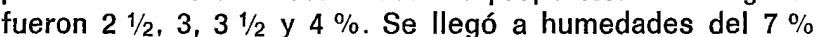
$y$ densidades de $2,18 \mathrm{~g} / \mathrm{cm}^{3}$. Se rompieron probetas con estas dosificaciones de cemento y humedad óptima a los 7 y a los 28 días en ambiente saturado de humedad, a $21^{\circ} \mathrm{C}$. Con las mismas proporciones de agua y cemento se hicieron ensayos de durabilidad, con ciclos de hielodeshielo y humedad-sequedad.

De acuerdo con los resultados de los ensayos se adoptó como dosificación óptima de cemento el 3,25\% con tolerancias de $\pm 0,25 \%$, con lo que puede llegarse a proporciones del $3 \%$ de conglomerante, que, aunque baja algo la resistencia, disminuye el peligro de fisuración.

Además de la dosificación de ligante, hay varios factores que tienen una influencia importante en las propiedades mecánicas de las capas de grava-cemento: el grado de compactación, la humedad y la granulometría. El tema está tratado ampliamente en una monografía francesa (3).

\section{los áridos}

El vigente Pliego contractual español (4) define la gravacemento como una mezcla íntima de áridos, cemento, agua $y$, eventualmente, adiciones, a la cual, convenientemente compactada, se le exigen determinadas condiciones.

Los áridos procederán de graveras o canteras cumpliendo las normales condiciones de limpieza y sometidos en parte a procesos de machaqueo. La fracción de piedra machacada será como mínimo el $40 \%$. Esta proporción 
exigiéndose el $100 \%$ si la vía es importante. La mayor rigidez de la mezcla a que dan lugar los elementos de machaqueo se traduce en una menor trabajabilidad o dificultad de extensión y compactación, pero el criterio de capacidad portante es dominante; la imbricación del árido de machaqueo aumenta notablemente la resistencia a flexotracción y todo ello es muy beneficioso para la estabilidad de la capa y de la sección estructural de que forma parte.

Respecto a la naturaleza del árido, no hay que confiar demasiado en el efecto de mejora que produce la incorporación del cemento. Esta no debe ser pretexto para una tolerancia excesiva en cuanto a contaminación, granulometría defectuosa, etc. Una uniformidad satisfactoria y una cota suficiente de resistencias mecánicas no pueden obtenerse más que con áridos de buena calidad procedentes de machaqueo y cribado, así como lavado cuando sea preciso. El mayor escrúpulo en la preparación de los áridos representa una de las más claras evoluciones en la técnica de la grava-cemento en los últimos años.

Se consideran dos granulometrías dependiendo del tamaño máximo del árido, que en las normas españolas corresponden a 20 y $37,5 \mathrm{~mm}$; se elige una u otra según los áridos disponibles a distancia económica y la capa que va a construirse. En Francia, según criterio reciente, estas dos granulometrías corresponden a las siguientes capas:

- bases de firmes asfálticos y capas de apoyo (se consideran como sub-bases) de firmes de hormigón de cemento; granulometría: $0 / 20 \mathrm{~mm}$;

- sub-bases de firmes asfálticos; granulometría: 0/31,5 milímetros $\left(^{*}\right)$

En la figura adjunta pueden verse los husos que corresponden a las dos granulometrías de referencia.

\section{GRANULOMETRIAS DE GRAVA - CEMENTO}

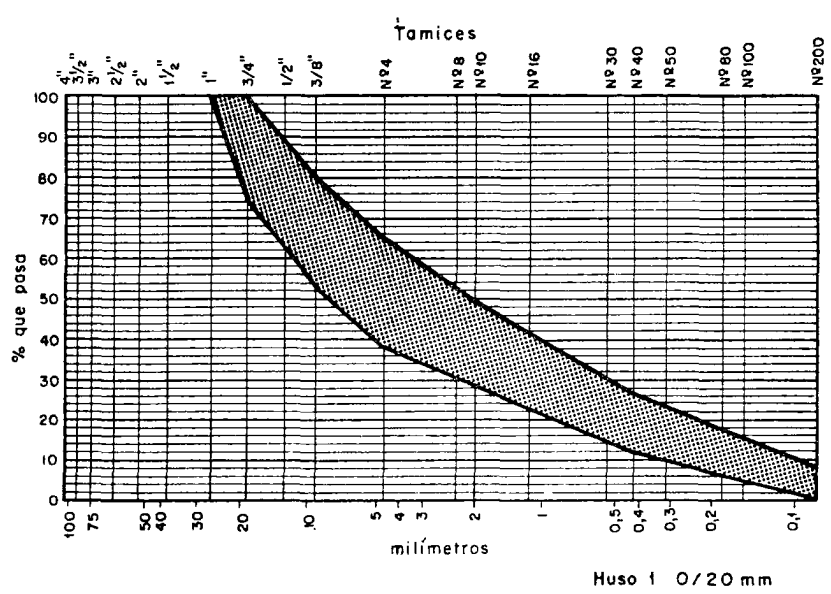

Se recomienda que la curva granulométrica que corresponde a la fórmula de trabajo sea lo más paralela posible a los límites del huso adoptado; pueden tolerarse ciertas insuficiencias en la fracción fina pensando que el defecto va a corregirse con el cemento.

La obtención de la granulometría requerirá una reconstitución del árido a partir de dos o tres acopios; en consecuencia, la planta tendrá dos o tres tolvas.

Cuando el yacimiento es un depósito aluvial suelen presentarse grandes variaciones en la proporción de arena. En el caso de que haya un exceso de arena, se separará antes del machaqueo la fracción inferior a $5 \mathrm{~mm}$. Al árido procedente del machaqueo, que será pobre en arena, se le incorporará la procedente de la separación previa.

No conviene llegar a fórmulas con exceso de arena - las correspondientes a la mitad superior de los husos- porque dan lugar a mezclas de menor resistencia a la compresión y con densidades más bajas.

El coeficiente de calidad medido por el ensayo de Los Angeles será, en todo caso, inferior a 40 . Actualmente hay una tendencia a mayores exigencias, aunque con carácter selectivo, en función del tráfico de la vía y la capa a que se destine la mezcla. En Francia, por ejemplo, se pide un coeficiente inferior a 35 ó 30 para capas de base o refuerzo, respectivamente, en tramos con más de 100 vehículos pesados al día.

Otras exigencias que debe cumplir el árido son las de plasticidad -determinada en la fracción que pasa por el tamiz $40(0,4 \mathrm{~mm})-$ y el contenido de materia orgánica.

Según las vigentes prescripciones españolas, los límites de Atterberg para la fracción definida cumplirán las condiciones siguientes: $L L<25$ e IP $<6$. Se limita también el equivalente de arena: EA $>30$. Con estas limitaciones se proscribe el empleo de áridos con exceso de finos plásticos, ya que no serían suficientemente neutralizadas con el cemento incorporado; además darían fácilmente lugar a dosificaciones incorrectas del conglomerante y a dispersiones en la resistencia mecánica de la capa.

El contenido de materia orgánica debe referirse a la mezcla en el momento de su extensión, incluyendo agua; no deberá pasar del $0,3 \%$, y siguiendo las recomendaciones francesas se determinará por el método del bicromato que figura en el Manual de Peltier (5).

\section{tipo y dosificación de cemento}

Normalmente se emplearán cementos portland ordinarios del tipo P-250, con un mínimo absoluto del $3 \%$ en la dosificación. En caso de que se desee prolongar el proceso constructivo por razones de equipo y organización. se puede emplear un cemento de fraguado lento.

La dosificación de cemento se ajustará ensayando proporciones medias en tres series de probetas compactadas con la humedad del Proctor modificado y curadas en condiciones normalizadas. La determinación del \% de cemento se hará en función de la resistencia mecánica y la durabilidad frente a las alteraciones higrotérmicas. 
En el cuadro adjunto figuran unas dosificaciones indicativas para las capas de base y sub-base:

\begin{tabular}{|c|c|c|}
\hline TIPO DE CEMENTO & $\begin{array}{c}\text { Bases de firme asfáltico } \\
\text { Sub-bases de firme de hormigón } \\
\text { Capas de refuerzo }\end{array}$ & $\begin{array}{c}\text { Sub-bases } \\
\text { de firme } \\
\text { asfáltico }\end{array}$ \\
\hline P-250 & $4,5 \%$ & $3,5 \%$ \\
P-350 & $3,5 \%$ & $3,0 \%$ \\
\hline
\end{tabular}

Como consecuencia de los ensayos de laboratorio, se exigirá la dosificación mínima con la que las probetas cumplan las siguientes condiciones:

- resistencia característica a la compresión simple entre 30 y $70 \mathrm{kp} / \mathrm{cm}^{2}$;

- pérdidas por cepillado después de los ciclos humectación-desecación y congelación-deshielo;

- este último sólo si el emplazamiento del tramo corresponde a zona fría, inferiores al $14 \%$ en peso.

\section{fabricación}

La mezcla debe hacerse en central por ser ésta la única manera de conseguir una distribución uniforme del cemento, lo que hay que cuidar especialmente en estas mezclas de baja dosificación. En consecuencia, se proscribe la mezcla in situ.

Las centrales que se emplean deberán adaptarse especialmente al tratamiento de grava-cemento, contando con dos o más tolvas dosificadoras de áridos, y dos silos de cemento $\left({ }^{*}\right)$ con capacidad superior a $50 \mathrm{t}$, mezcladora de ejes gemelos y dosificador de agua.

Las normas francesas recomiendan instalaciones de tipo continuo. El rendimiento de la central dependerá del ritmo de puesta en obra y a éste deberá adaptarse.

En todo caso se cuidará especialmente de que tanto las palas, dumpers y otros vehículos cargadores de áridos y la flota de camiones de transporte de la mezcla a obra se adapten al ritmo de trabajo, con el fin de evitar al máximo las interrupciones de la central.

La capacidad de los silos corresponderá al rendimiento de producción de la central y se cuidará de que el nivel de los áridos se mantenga lo más constante posible. Se recomienda la instalación de un vibrador que actúe sobre las paredes del silo y evite que la arena, sobre todo cuando está húmeda, forme bóveda e impida al flujo normal.

\section{puesta en obra}

La puesta en obra de las capas de grava-cemento está condicionada por el breve período en que deben realizarse todas las operaciones. En efecto, en condiciones normales de temperatura, y para el tipo de cemento que suele emplearse, este período es del orden de 2 horas.
Se impone, en consecuencia, una buena organización, con equipos suficientes y programa estricto de trabajos.

Como los espesores de las bases o sub-bases no superarán los 20 ó $25 \mathrm{~cm}$, la extensión se hará de una sola vez.

Para el período consignado se recomienda un ritmo de obra de $100 \mathrm{~m}$. I./hora, que en calzadas de $7 \mathrm{~m}$ supone unas $300 \mathrm{t} /$ hora.

En la autopista Barcelona-Granollers se fabricó la gravacemento en una planta con rendimiento máximo de $350 \mathrm{t} /$ hora, con hormigonera de $5 \mathrm{~m}^{3}$. En el tramo Mongat-Mataró la planta fue de $200 \mathrm{t} /$ hora.

En la extensión se emplean cada vez más las máquinas dotadas de sistemas de nivelación automática y dispositivo controlador de pendientes. El control de nivelación se efectúa con palpadores que se apoyan sobre un alambre tenso que sirve de guía de referencia.

En el caso de que la capa sea la sub-base de un pavimento de hormigón hidráulico en el que se vaya a emplear la máquina de encofrados deslizantes, se utiliza ésta también para la grava-cemento. En la autopista París-Lyon, tramo de Athie a Pouilly-en-Auxois (Departamento de Dijon), de $57,900 \mathrm{~km}$ de longitud, se empleó, por esta razón, la máquina de encofrados deslizantes para la sub-base cuyo espesor era de 15 centímetros.

No vamos a descubrir aquí la gran importancia de la compactación, cuyo suficiente grado respecto a una densidad-patrón debe asegurarse para evitar posteriores asientos diferenciales por efecto de la frecuencia y grandes cargas de los vehículos pesados.

Los compactadores que se emplean normalmente para las capas de grava-cemento son los rodillos neumáticos y los rodillos vibratorios.

En los rodillos neumáticos, las caracteristicas que influyen en las posibilidades de conseguir una densidad dada son el peso, la presión de inflado y el espesor de la capa. El peso por rueda será de 2 ó $4 \mathrm{t}$-dependiendo de que el árido sea rodado o de machaqueo en su totalidady la presión será superior a $5 \mathrm{kp} / \mathrm{cm}^{2}$.

\section{GRANULOMETRIAS DE GRAVA - CEMENTO}

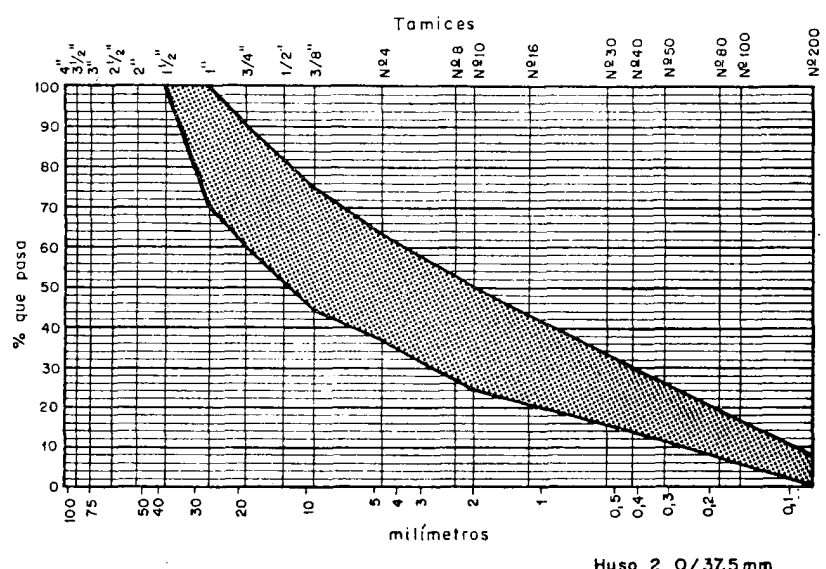

Huso $20 / 37,5 \mathrm{~mm}$

(*) Son precisos dos silos, ya que no debe usarse nunca un sllo que se esté llenando. 
En los rodillos vibratorios influye el peso y la frecuencia, ligada ésta a la granulometría del árido. Se recomiendan rodillos de peso superior a 4 toneladas.

Las nuevas recomendaciones francesas, interesantes en este punto, fijan los siguientes equipos para dos ritmos de obra diferentes:

\section{Volumen de mezcla empleado}

$$
\begin{aligned}
& <1.500 \mathrm{t} / \text { día } \\
& >1.500 \mathrm{t} / \text { día }
\end{aligned}
$$

\section{Rodillo vibratorio pesado}

- uno y 3 pasadas

- uno y 3 pasadas

\section{Rodillo neumático pesado}

uno y 15 a 20 pasadas

- dos o más y 15 a 20 pasadas

Estas son normas orientadoras, pero en las obras importantes conviene establecer tramos testigos que permitan comprobar la eficacia de distintos tipos de rodillo y definir el número de pasadas para obtener la densidad especificada en el Pliego contractual. Estos tramos tendrán como mínimo $200 \mathrm{~m}^{2}$. Se fija normalmente un $98 \%$ del Proctor modificado, a la que debe llegarse en el $95 \%$ de las determinaciones.

La experiencia ha mostrado que, para unos materiales y una fórmula de trabajo dada, las variaciones en el grado de compactación influyen considerablemente en la resistencia mecánica de la capa. Por ejemplo, el bajar del 100 al $95 \%$ del Proctor modificado supone reducciones de resistencia a la compresión que oscilan entre el 20 y el $45 \%$.

\section{CORRELACION DE DENSIDADES - RESISTENCIAS}

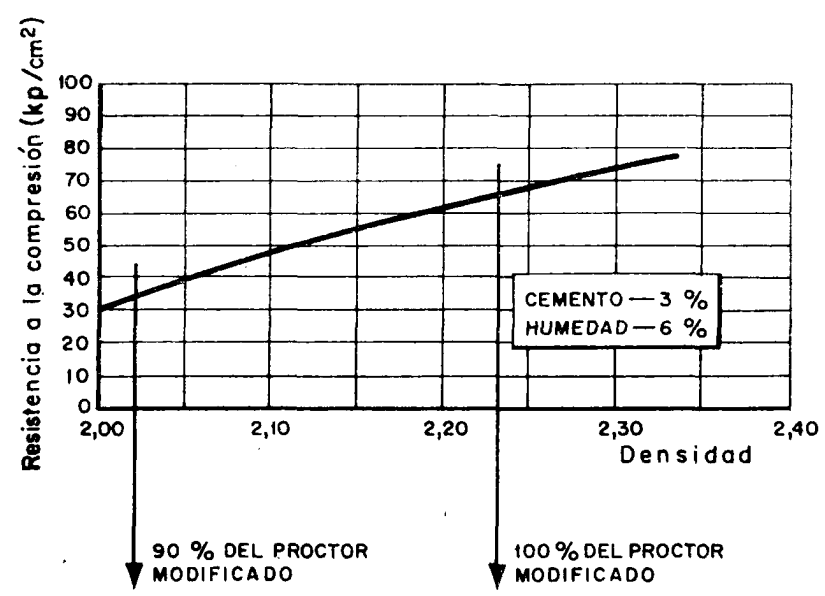

Según ha mostrado la experiencia para unos materiales y una fórmula de trabajo dada, el grado de compactación, o sea la densidad obtenida en obra, influye notablemente en la resistencia mecánica de la capa. Como la resistencia a la compresión se reduce a la mitad.
Después de la compactación se llevará a cabo una regularización o perfilado con niveladora, o sea, el llamado acabado, con el que se deja la capa de acuerdo con las cotas $y$ pendientes que figuran en los planos.

Posteriormente, se aplicará un riego de curado con emulsión catiónica muy diluida, de modo que el betún residual sea del orden de $0,3 \mathrm{~kg} / \mathrm{m}^{2}$. Este riego se aplicará antes de transcurridas 8 horas después del acabado; en días de sol este período se reducirá a 4 horas. Sobre la emulsión se extienden gravilla o arena limpia.

La apertura al tráfico depende de la intensidad de vehículos pesados. Si el número de éstos es inferior a 100 , se puede dar paso una hora después de terminada la compactación. Si la intensidad de éstos es superior a 100 al día, hay que esperar como mínimo 12 horas.

Las capas de grava-cemento por destinarse en general a vías de tráfico pesado se cubrirán con un revestimiento asfáltico, normalmente una mezcla en caliente. Cuando se proyecta un tratamiento superficial - suele ser esta solución temporal para una primera fase- será doble, con betún fluido RC-4 o emulsión catiónica al $60 \%$. En ambos casos el betún residual será del orden de $2,5 \mathrm{~kg} / \mathrm{m}^{2}$.

Los espesores de mezcla asfáltica varían entre 10 y $20 \mathrm{~cm}$, según se disponga sólo el pavimento (capa de rodadura + capa intermedia) o también una capa de base. Los problemas de transmisión de las grietas de retracción de la grava-cemento a la superficie del pavimento han motivado la tendencia a espesores asfálticos mayores.

Por ejemplo, en los tramos citados de las autopistas catalanas se han extendido sobre la capa de grava-cemento de $20 \mathrm{~cm}$ tres capas asfálticas de 12,5 y $4 \mathrm{~cm}$, pero se prevé aumentar este espesor de los 21 a $25 \mathrm{~cm}$, extendiendo otra capa de $4 \mathrm{~cm}$ después de algún tiempo de servicio.

Las mezclas que se proyectan para estas capas deberán ser de alto módulo de rigidez (betunes duros 60-80, ó 40-60 si la obra está en zona cálida, árido duro y en su totalidad de machaqueo, al menos en la fracción gruesa, etc.) para evitar deformaciones por el liamado efectoyunque, en condiciones desfavorables de altas temperaturas ambiente y lenta aplicación de las cargas pesadas (rampas, zonas de frenado, etc.).

\section{un ejemplo español}

Concluimos el presente trabajo reseñando las características esenciales de una obra española, la base de gravacemento de la Variante de Torrejón de Ardoz, tramo de autopista en la $\mathrm{CN}-\mathrm{Il}$ de Madrid a Francia por Barcelona, con lo que se ha sustituido la travesía del citado pueblo.

Esta base corresponde a la capa de asiento del firme de hormigón hidráulico con losas no armadas de $25 \mathrm{~cm}$ de espesor. El proyecto original llevaba base granular, pero se sustituyó por grava-cemento para conseguir una mayor 
resistencia y evitar peligrosos fenómenos de pumping o surgencia.

Tanto la sub-base también estabilizada con cemento como la base de $15 \mathrm{~cm}$ de espesor se describen en la publicación de referencia (6). Los materiales, equipos y exigencias son los que se definen a continuación:

Aridos: Grava silicea de machaqueo y arena natural, también silícea, en proporciones del $50 \%$.

Granulometría: Tamaño máximo, $2,5 \mathrm{~cm}$. Encaja en el huso propuesto por el Laboratorio Regional de Madrid que corresponde al huso francés.

Plasticidad: N.P.

Equivalente de arena: Mayor que 80.

Contenido de cemento: $6 \%$ del tipo P-250.

\section{Equipos}

Fabricación: Planta "Barber-Greene" de 140/180 t/hora. Extensión: Una extendedora "Barber-Greene" de aglomerado.

Compactación: Un rodillo liso $(5$ t). Un rodillo tándem $(12 \mathrm{t}$ ). Un rodillo vibratorio de $1,4 \mathrm{t}$ (bordes).

Grado de compactación: Superior al $95 \%$ del Proctor mo dificado.

Resistencia a compresión: A los 7 días, $45 \mathrm{kp} / \mathrm{cm}^{2}$. Se exigian $35,7 \mathrm{kp} / \mathrm{cm}^{2}$ en el Pliego contractual.

Curado: Riego con emulsión asfáltica $\left(0,7 \mathrm{~kg} / \mathrm{m}^{2}\right)$.

Producción: 120 t/hora.
En el presente trabajo hemos pretendido hacer una exposición de las actuales normas y tendencias en la técnica de las capas de grava-cemento. Su utilización cada vez más frecuente, tanto en firmes nuevos como en el refuerzo de los que tienen una estabilidad precaria, estimamos justifica esta vuelta de horizonte sobre el tema, a efectos singularmente divulgadores, como una primera información para proyectistas y constructores que en el futuro consideren esta solución conveniente en muchos casos y sobradamente sancionada por la experiencia nacional y extranjera.

\section{referencias bibliográficas}

(1) Directive pour la realisation des assises de chaussées en graves-ciment. Ministère de l'Équipement et du Logement. Service d'Études Techniques des Routes et Autoroutes (SETRA), Paris, Mars 1969.

(2) LLAMAZARES, O.: La técnica de los afirmados en la V Reunión de la Federación Internacional de Carreteras, Boletín de Información del M. O. P. núm. 109.

(3) BONNOT, J.: Les renfforcements de chaussées en graves-ciment. Bulletin de Liaison des Laboratoires Routiers. Special H. Paris, 1967.

(4) Pliego de prescripciones técnicas generales para obras de carreteras y puentes. (En revisión). Dirección General de Carreteras, M.O.P. Madrid, 1965.

(5) PELTIER, M.: Manuel du Laboratoire Routier. Editions Dunod. Paris, 1969.

(6) SOLER BOIX, R.: Estabilizaciones con cemento. Curso especial de Carreteras. "Los materiales". Madrid, octubre 1968

\section{résumé}

L'emploi de gravier-ciment pour les revêtements routiers

Olegario Llamazares Gómez, Dr. ingénieur des Ponts et Chaussées

Les couches de gravier-ciment sont employées, depuis plusieurs années, pour les revêtements routiers, remplaçant les bases granulaires pou les troncons où le trafic exige des sections structurales à haute résistance.

L'expérience conseillé l'évolution de cette technique pour parvenir à l'élaboration de cou ches de meilleure qualité et garantio avec une sélection plus soignée des agrégats, des dosages, du type de ciment et des conditions de fabrication, mise en oeuvre et cure. Dans cet article, l'auteur rend compte des tendances actuelles dans ce domaine et indique les exigences des Cahiers des charges.

\section{summary}

The use of gravel-cement in road surfacing

Dr. Olegario Llamazares Gómez, Civil Engineer

Layers of gravel-cement have been used for several years in road surfacing replacing the several years in road surfacing replacing the makes high-strength structural sections necessary.

Experience has brought about the evolution of this technique so as to obtain higher quality layers of greater guarantee with a more careful
selection of the aggregates with respect to selection of the aggregates with respect to granulometry and form, batching and cement type and manufacturing conditions, implementation and curing. This article reports on th ing the requirements of contract specifications.

\section{zusammenfassung}

Die Verwendung von Zementkies für Strassendecken

Dr. Olegario Llamazares Gómez, Tiefbauningenieur

Zementkiesschichten werden schon seit Jahren eingesetzt für Strassendecken an Stelle der körnigen Unterlagen in jenen Abschnitten, wo de Vekehr eine besonders widerstandsfähige Struk tur erforderlich macht.

Die Praxis liess eine Weiterentwicklung dieser Technik ratsam erscheinen, um so Schichten besserer Qualität und Garantie zu erzielen be sorgfältigerer Auswahl der Zuschläge hinsichtlich ihrer Körnungsgrösse und Form, Dosierungen und Art des Zements, Herstellungsbedingungen, Aufbringung und Nachbehandlung. Der vorliegende Artikel informiert über die heutigen Tendenzen auf allen diesen Gebieten und gibt Hinweise auf die Anforderungen in den Vertragsbedingungen. 